Supporting Information

\title{
Photoinduced Reduction of Nitroarenes Using a Transition Metal-Loaded Silicon Semiconductor under Visible Light Irradiation
}

\author{
Ken Tsutsumi, ${ }^{*}{ }^{\dagger}$ Fumito Uchikawa, ${ }^{\ddagger}$ Kentaro Sakai, ${ }^{\S}$ and Kenji Tabata** \\ 'Department of Chemistry, Graduate School of Science and Engineering, Tokyo Metropolitan \\ University \\ 1-1 Minami Osawa, Hachioji, Tokyo, 192-0364, Japan \\ ${ }^{*}$ Department of Applied Chemistry, Faculty of Engineering, University of Miyazaki \\ 1-1, Gakuen-Kibanadai-Nishi, Miyazaki, 889-2155, Japan \\ ${ }^{\S}$ Center for Collaborative Research \& Community Cooperation, University of Miyazaki \\ 1-1, Gakuen Kibanadai-Nishi, Miyazaki, 889-2155, Japan. \\ Correspondence to: tsutsumi@tmu.ac.jp and kenjt@cc.miyazaki-u.ac.jp
}

\section{Contents}

$\begin{array}{ll}\text { 1. Materials and reagents } & \mathrm{S} 2\end{array}$

2. Preparation of $\mathrm{M} / \mathrm{Si}(\mathrm{M}=\mathrm{Pd}, \mathrm{Pt}, \mathrm{Ru}) \quad \mathrm{S} 2$

3. $\mathrm{N}_{2}$ adsorption-desorption analysis of $\mathrm{M} / \mathrm{Si}(\mathrm{M}=\mathrm{Pd}, \mathrm{Pt}, \mathrm{Ru}) \quad \mathrm{S} 2$

4. X-ray powder diffraction (XRD) analysis of $\mathrm{M} / \mathrm{Si}(\mathrm{M}=\mathrm{Pd}, \mathrm{Pt}, \mathrm{Ru}) \quad \mathrm{S} 4$

5. Scanning electron microscope (SEM) images of $\mathrm{M} / \mathrm{Si}(\mathrm{M}=\mathrm{Pd}, \mathrm{Pt}, \mathrm{Ru}) \quad \mathrm{S} 5$

6. Transmission electron microscope (TEM) images of $\mathrm{M} / \mathrm{Si}(\mathrm{M}=\mathrm{Pd}, \mathrm{Pt}, \mathrm{Ru}) \quad \mathrm{S} 6$

7. General procedure for photocatalytic reduction of nitroarenes in the presence of $\quad$ S8 metal-loaded Si semiconductors 


\section{Materials and reagents.}

Silicon (Si 99.999\%, Aldrich Chemical Co.; Si, Kojundo Chemical Laboratory, Ltd.; Si crystalline 99.999\%, Alfa Aesar) was used as a semiconductor. Dispersions of Pd, Pt and Ru nanoparticles in divinylbenzene polymer (Wako Pure Chemical Industries, Ltd.) were used as the metal source to prepare metal-loaded Si semiconductors. $\mathrm{HCOOH}$ and $\mathrm{HF}$ (46\%) were also sourced from Wako Pure Chemical Industries, Ltd. Nitrobenzene (1a), 4-, 3- or 2-nitroacetophenone (1b-d), 4-nitrobenzonitrile (1e), 3-nitrostyrene (1f), and 4-bromonitrobenzene (1g) were used as reactants and purchased from Wako Pure Chemical Industries, Ltd. Palladium on activated carbon (5 wt\% $\mathrm{Pd} / \mathrm{C}$, Wako Pure Chemical Industries, Ltd.) was used as a catalyst for the reduction of nitrobenzene. These commercially available reagents were used without further purification.

\section{Preparation of $\mathrm{M} / \mathrm{Si}(\mathbf{M}=\mathbf{P d}, \mathbf{P t}, \mathbf{R u})$.}

Metal-loaded semiconductors were prepared via an impregnation method. Three kinds of silicon (as listed above) were used as the support. Si powder ( $2 \mathrm{~g})$ was stirred in aqueous HF (46\%, 40 $\mathrm{mL}$ ) for $40 \mathrm{~min}$ to decrease the size of silicon particles (increase the surface area of silicon particles). The sample was rinsed three times with deionised water, twice with each methanol and acetone and was dried under atmospheric conditions for $24 \mathrm{~h}$. The obtained powder $(2.0 \mathrm{~g})$, deionised water $(20 \mathrm{~mL})$ and a calculated amount of metal nanoparticle (Pd, Pt or $\mathrm{Ru})$ dispersion were mixed to produce $5 \mathrm{wt} \%$ silicon after impregnation. The mixture was dried on a hot plate at $80{ }^{\circ} \mathrm{C}$ and the resulting product was heat-treated under a stream of argon gas at $150{ }^{\circ} \mathrm{C}$ for $1 \mathrm{~h}$ and $400{ }^{\circ} \mathrm{C}$ for $15 \mathrm{~min}$. The sample was rinsed with deionised water, ethanol, diethyl ether, $n$-hexane and acetone and dried at $80^{\circ} \mathrm{C}$. The noble metal content in the samples was calculated by Inductively Coupled Plasma Mass Spectrometry (ICP-MS) measurement (ICPM-8500, Shimadzu).

\section{3. $\mathrm{N}_{2}$ adsorption-desorption analysis of $\mathrm{M} / \mathrm{Si}(\mathrm{M}=\mathbf{P d}, \mathrm{Pt}, \mathrm{Ru})$.}

The Brunauer-Emmett-Teller (BET) surface areas of the samples were measured by nitrogen adsorption at $-196{ }^{\circ} \mathrm{C}$ (BELSORP-miniII, BEL Japan, Inc.). Prior to nitrogen physisorption, the samples were degassed under vacuum at $200{ }^{\circ} \mathrm{C}$ for $2 \mathrm{~h}$ (Figure S1). The Brunauer-Emmett-Teller (BET) approach was used to calculate the specific surface areas using the adsorption data over the relative pressure range from $0.05-0.35$ (Table S1). 

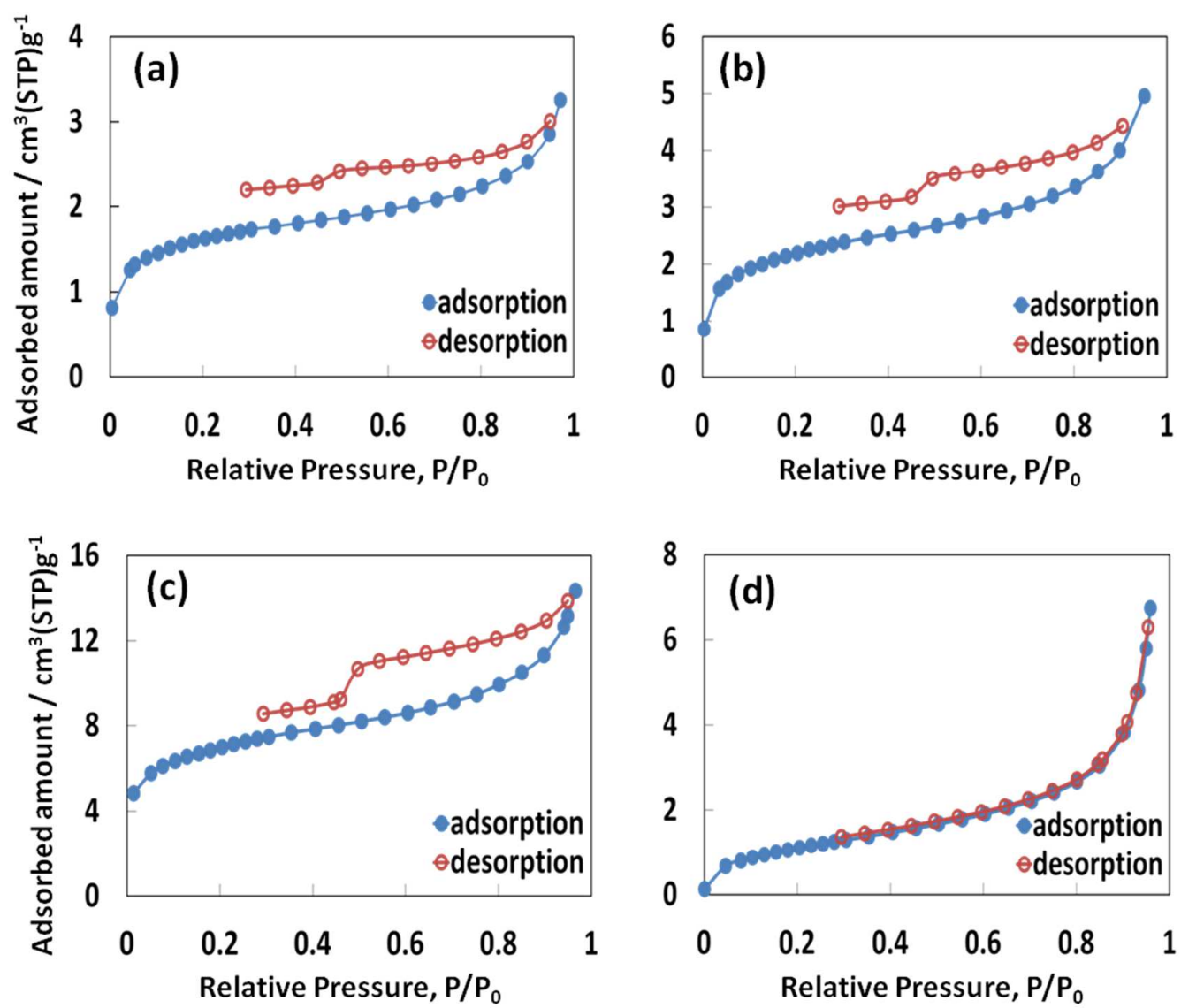

Figure S1. $\mathrm{N}_{2}$ adsorption-desorption isotherms for (a) Pd/Si catalyst (Si 99.999\%, Aldrich Chemical Co.), (b) Pt/Si catalyst (Si 99.999\%, Aldrich Chemical Co.), (c) Ru/Si catalyst (Si 99.999\%, Aldrich Chemical Co.), and (d) Si catalyst (Si 99.999\%, Aldrich Chemical Co.). The samples were first degassed under vacuum at $200^{\circ} \mathrm{C}$ for $2 \mathrm{~h}$.

Table S1. BET surface areas of the catalysts.

\begin{tabular}{cc}
\hline catalyst & BET surface area $\left(\mathrm{m}^{2} \mathrm{~g}^{-1}\right)$ \\
\hline Pd/Si catalyst (Si 99.999\%, Aldrich Chemical Co.) & 6.0 \\
Pt/Si catalyst (Si 99.999\%, Aldrich Chemical Co.) & 8.0 \\
Ru/Si catalyst (Si 99.999\%, Aldrich Chemical Co.) & 25.4 \\
Si catalyst (Si 99.999\%, Aldrich Chemical Co.) & 3.6 \\
Si catalyst (Si 99.999\%, Aldrich Chemical Co. $)^{a}$ & 4.2 \\
\hline
\end{tabular}

\footnotetext{
${ }^{a}$ After washing with HF.
} 


\section{X-ray powder diffraction (XRD) analysis of $\mathrm{M} / \mathrm{Si}(\mathrm{M}=\mathrm{Pd}, \mathrm{Pt}, \mathrm{Ru})$.}

X-ray diffraction (XRD) measurements were carried out on a PANalytical X'Pert Pro Multipurpose Diffractometer scanning from $20^{\circ}$ to $70^{\circ}(2 \theta)$ at a rate of $0.5^{\circ} \min ^{-1}$ using a $\mathrm{Cu}-\mathrm{K} \alpha$ radiation $(\lambda=0.15418 \mathrm{~nm})$ source. The applied voltage and current were $45 \mathrm{kV}$ and 40 $\mathrm{mA}$, respectively.
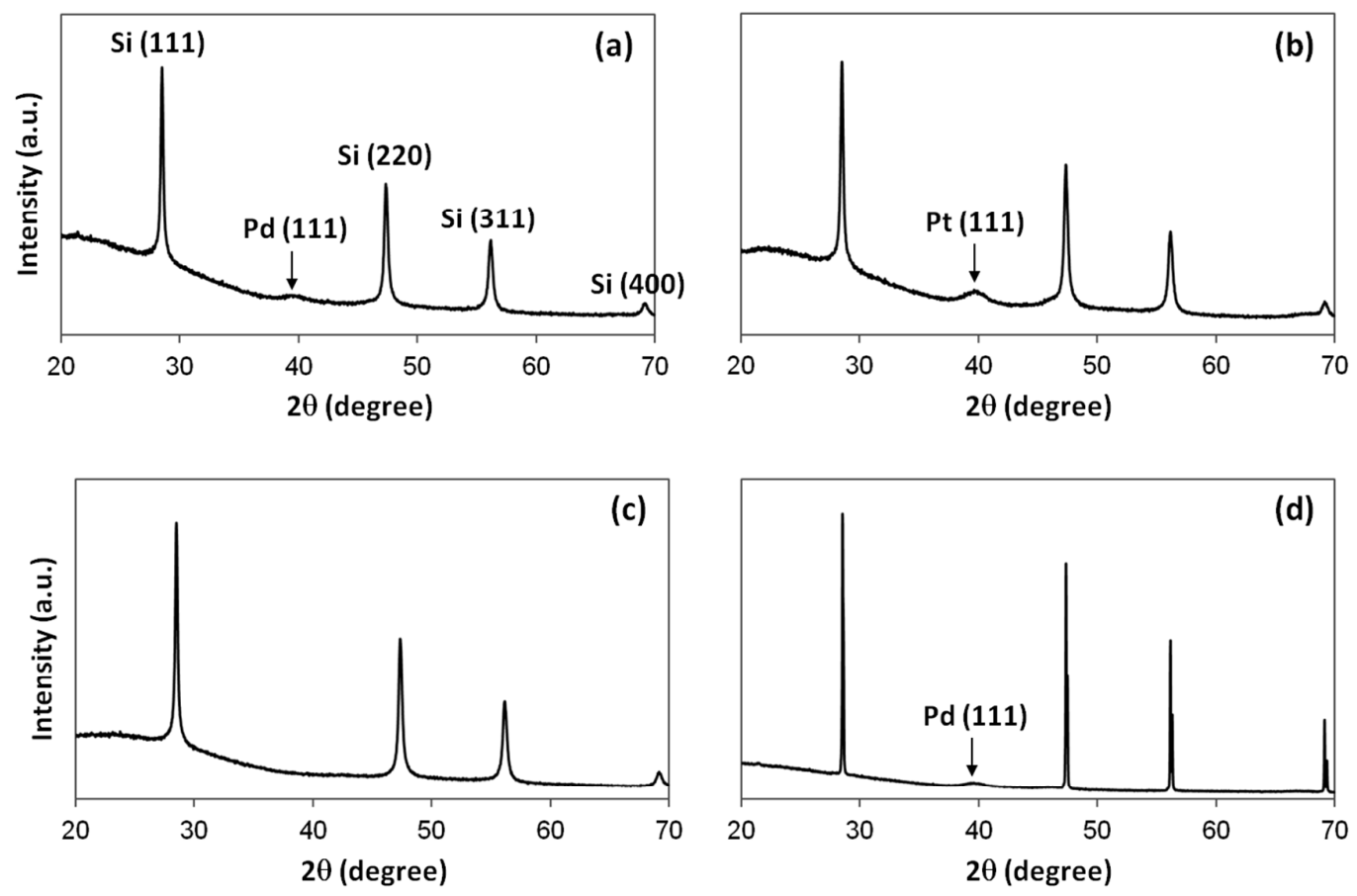

Figure S2. XRD patterns for (a) Pd/Si catalyst ( $\mathrm{Si} 99.999 \%$, Aldrich Chemical Co.), (b) $\mathrm{Pt} / \mathrm{Si}$ catalyst (Si 99.999\%, Aldrich Chemical Co.), (c) Ru/Si catalyst (Si 99.999\%, Aldrich Chemical Co.), and (d) Pd/Si catalyst (Si crystalline 99.999\%, Alfa Aesar). 


\section{Scanning electron microscope (SEM) images of $\mathrm{M} / \mathrm{Si}(\mathbf{M}=\mathbf{P d}, \mathrm{Pt}, \mathrm{Ru})$.}

Three-dimensional images of the surface of the metal-supported photocatalysts were observed using a scanning electron microscope (SEM) with S-5500 (Hitachi Ltd.). The acceleration voltage of the electrons was $20.0 \mathrm{kV}$.
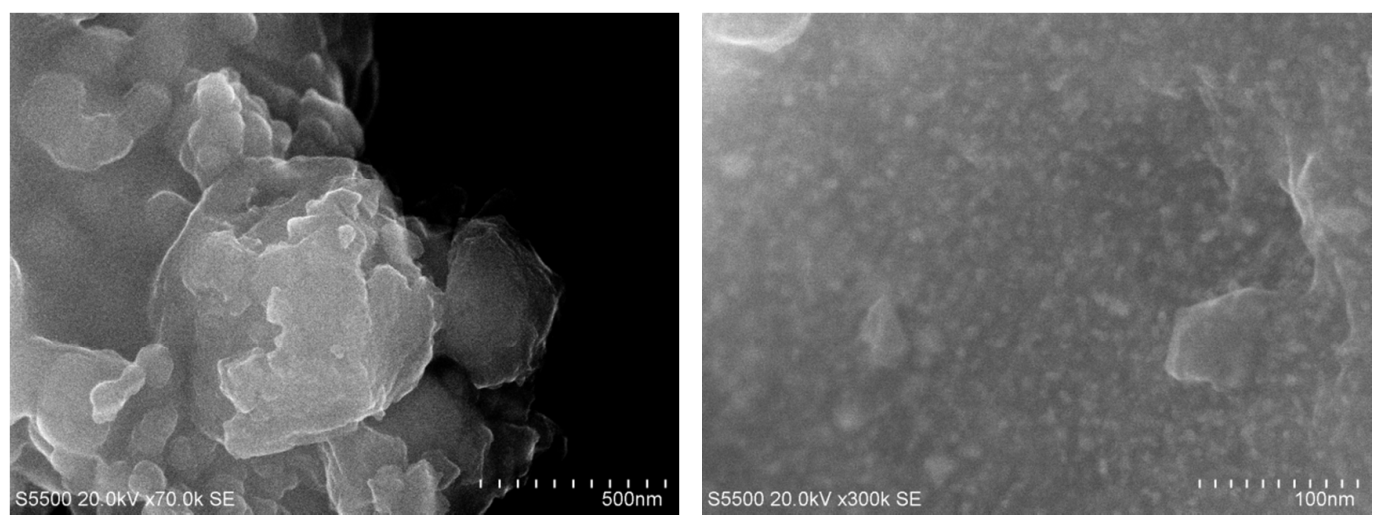

Figure S3. SEM images of the Pd/Si catalyst (Si $99.999 \%$, Aldrich Chemical Co.).
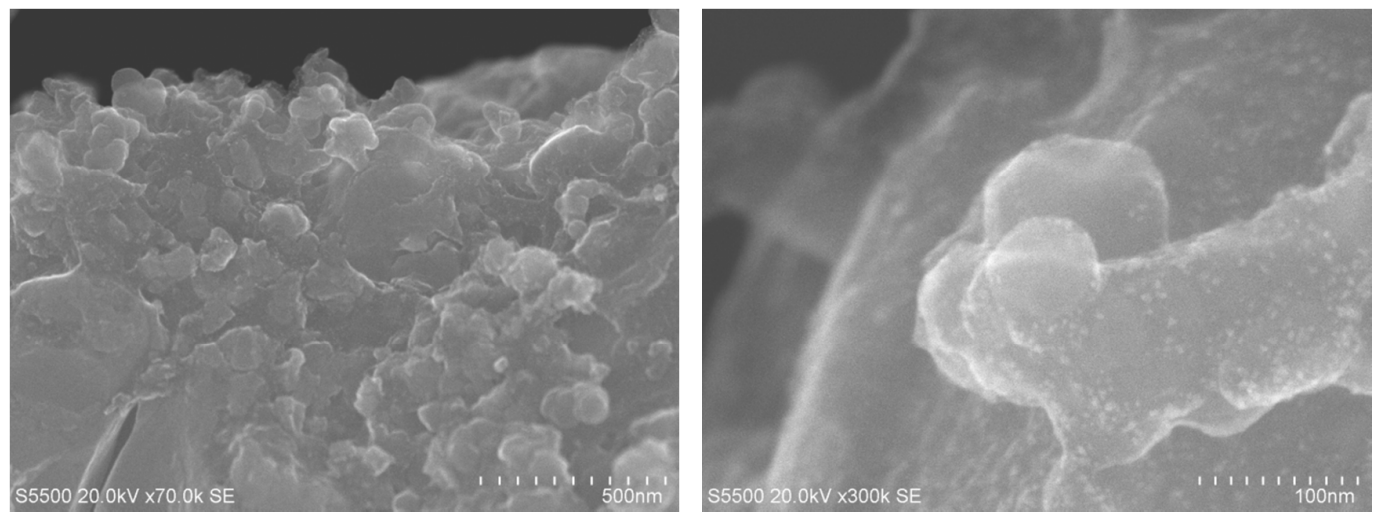

Figure S4. SEM images of the Pt/Si catalyst (Si 99.999\%, Aldrich Chemical Co.).
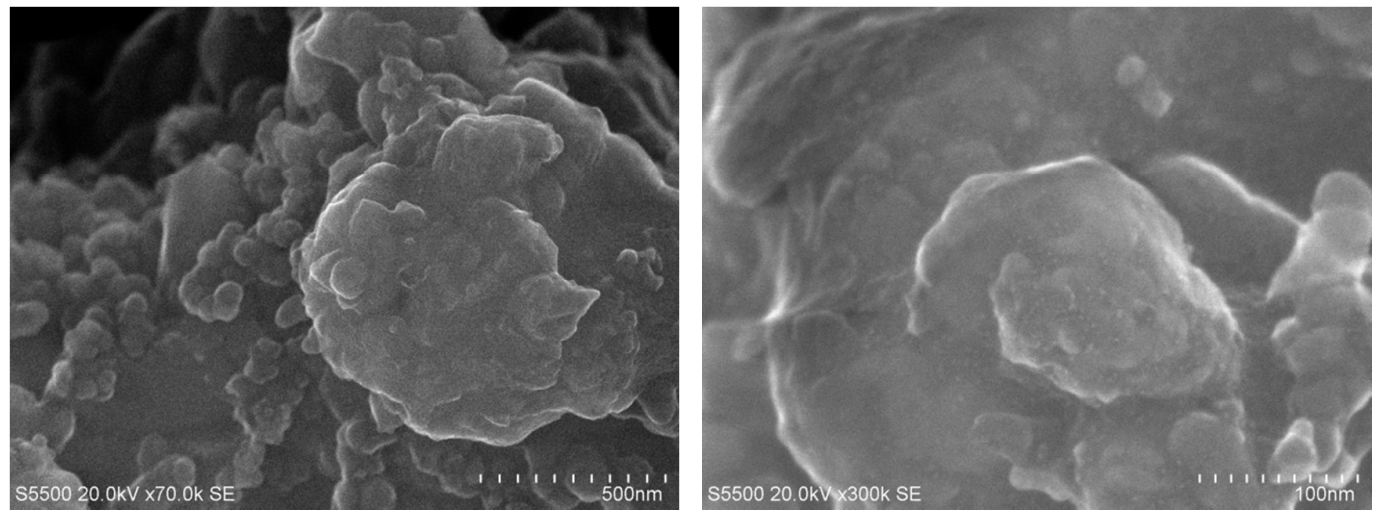

Figure S5. SEM images of the Ru/Si catalyst (Si 99.999\%, Aldrich Chemical Co.). 

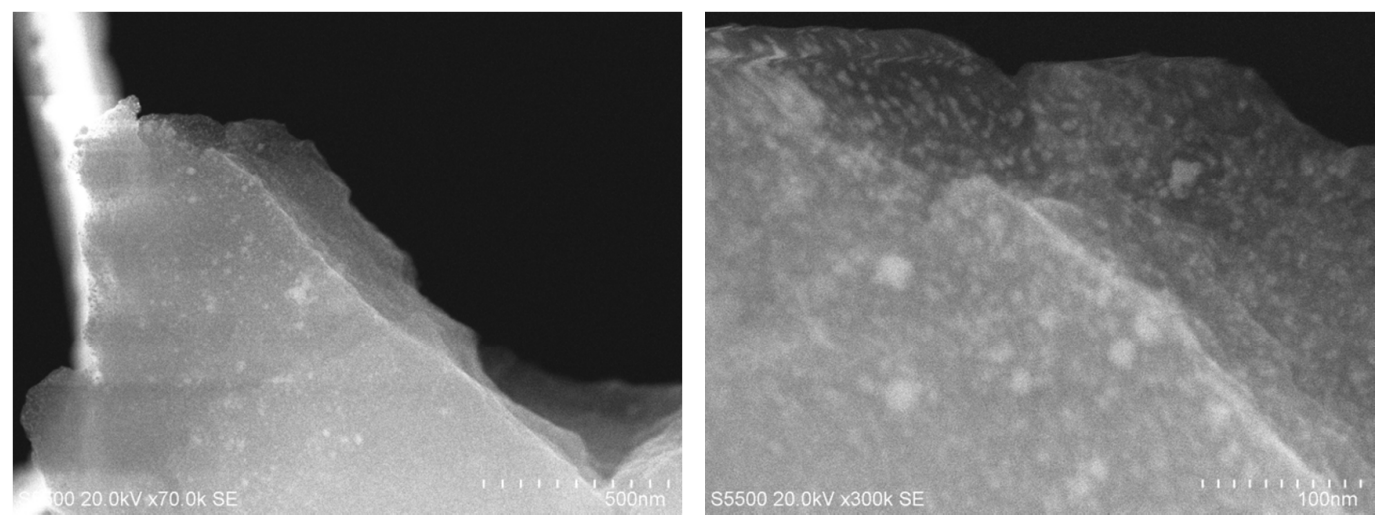

Figure S6. SEM images of the Pd/Si catalyst (Si crystalline 99.999\%, Alfa Aesar).

\section{Transmission electron microscope (TEM) images of $M / \mathrm{Si}(\mathrm{M}=\mathrm{Pd}, \mathrm{Pt}, \mathrm{Ru})$.}

Three-dimensional images of the surface of the metal-supported photocatalysts were observed using a transmission electron microscope (TEM) with S-5500 (Hitachi Ltd.). The acceleration voltage of the electrons was $200.0 \mathrm{kV}$.

The photocatalyst morphology and particle size of the metal-supported photocatalysts were investigated using a TEM, (JEOL, JEM-2010MX) under operating voltages of $15 \mathrm{kV}$ and $200 \mathrm{kV}$, respectively.
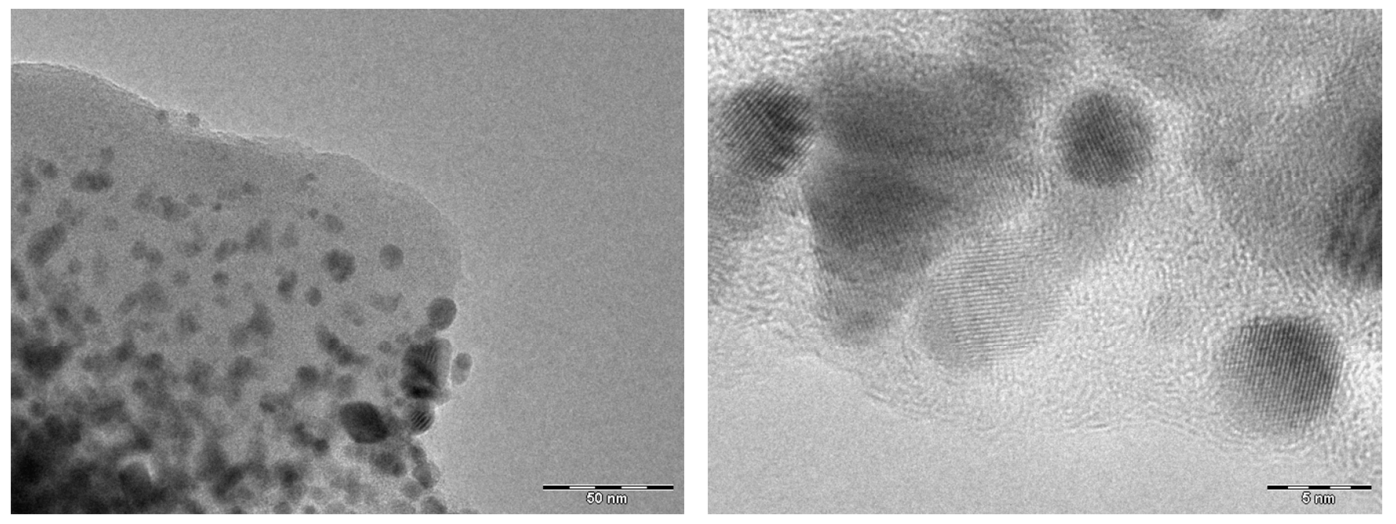

Figure S7. TEM images of the Pd/Si catalyst (Si 99.999\%, Aldrich Chemical Co.). 

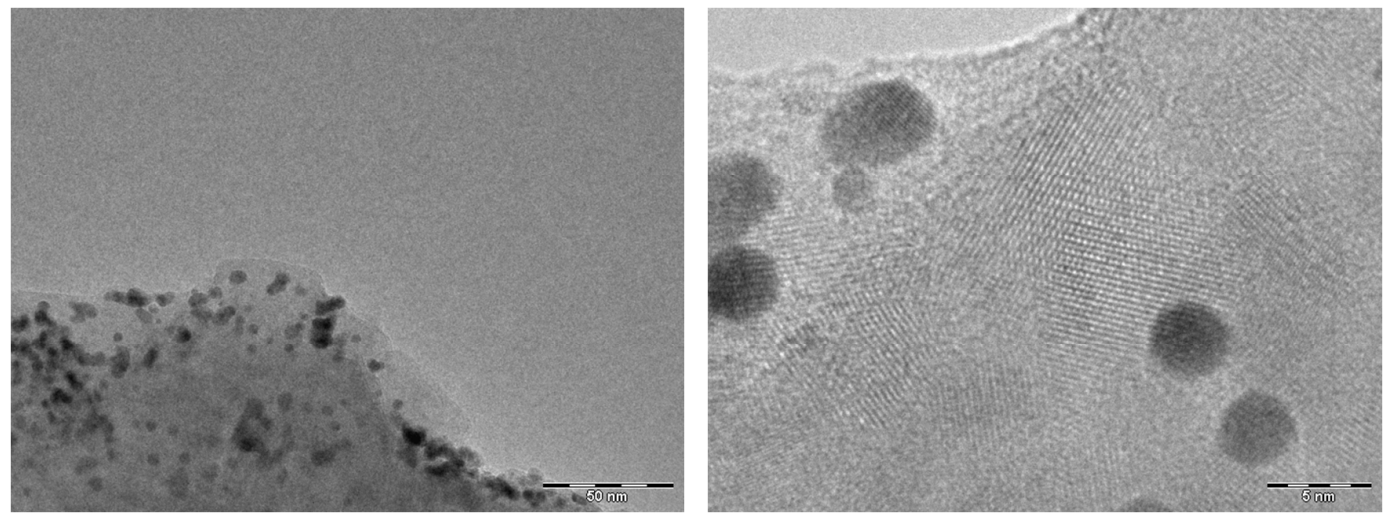

Figure S8. TEM images of the Pt/Si catalyst (Si 99.999\%, Aldrich Chemical Co.).
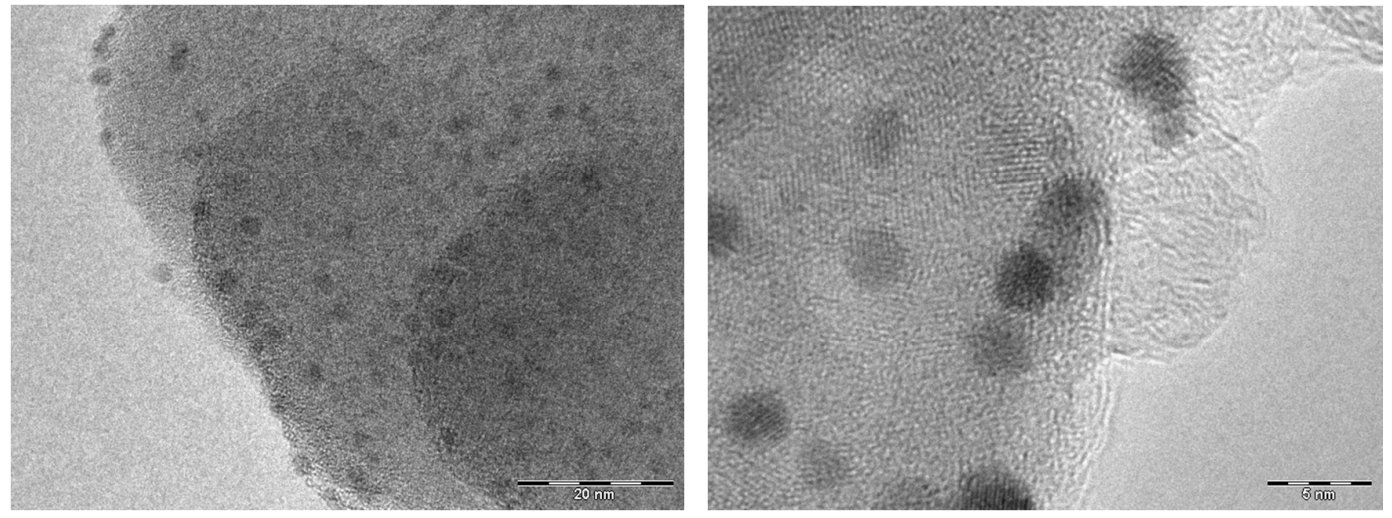

Figure S9. TEM images of the Ru/Si catalyst (Si 99.999\%, Aldrich Chemical Co.).
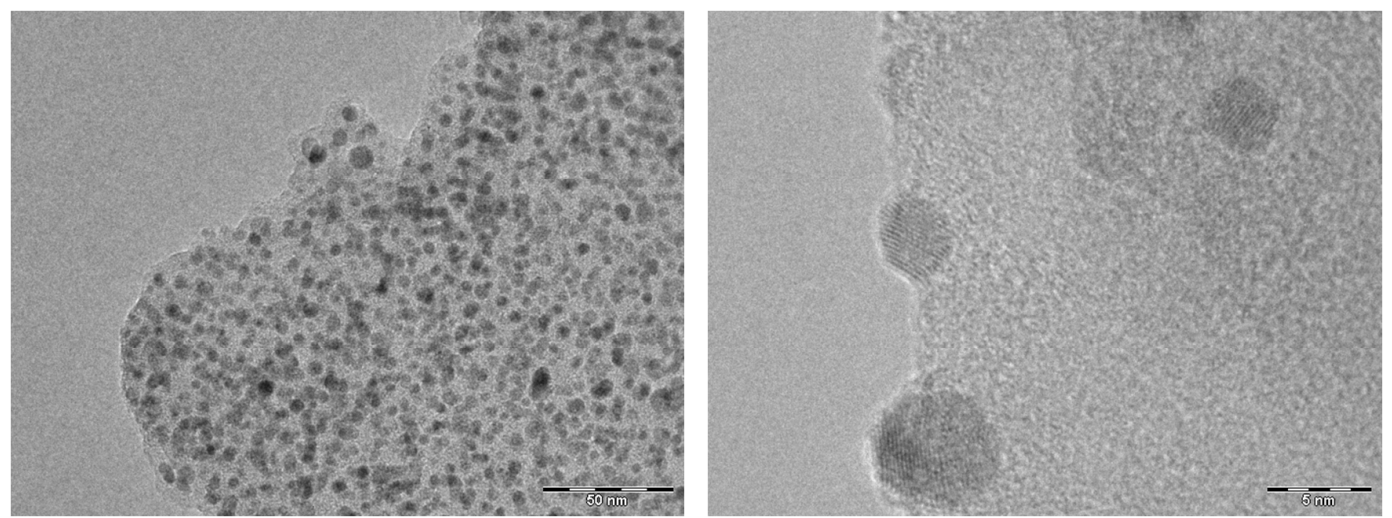

Figure S10. TEM images of the Pd/Si catalyst (Si crystalline 99.999\%, Alfa Aesar). 


\section{General procedure for photocatalytic reduction of nitroarenes in the presence of}

metal-loaded Si semiconductors.

A suspension of $\mathrm{Pd} / \mathrm{Si}(25 \mathrm{mg})$ in $\mathrm{H}_{2} \mathrm{O} / 2$-propanol (10:90) (4.6 mL) was placed in a Pyrex test tube (14.5 mm inner diameter (I.D.), $16.5 \mathrm{~mm}$ outer diameter). After deaeration of the suspension by bubbling pure nitrogen, nitrobenzene $1 \mathrm{a}(6.2 \mathrm{mg}, 0.050 \mathrm{mmol})$ and formic acid $(8.3 \mathrm{mg}, 0.18 \mathrm{mmol})$ were added. The sealed test tube was positioned in a quartz bath and the temperature of the bath was controlled with a chiller (Figure S11). The suspension was stirred and photoirradiation was carried out using a xenon lamp (300 W, LX-300F, PE ILS) as the light source equipped with an UV cut-off filter (SC0750, Asahi Spectra Co., Ltd). After 3 h, the resulting mixture was filtered through a 0.2 $\mu \mathrm{m}$ Millipore filter to remove the insoluble powder. In the case of catalyst recyclability tests, the reaction suspension was centrifuged and the supernatant solution was removed. The remaining solid was rinsed with deionised water, ethanol, diethyl ether, $n$-hexane and acetone and dried at $80{ }^{\circ} \mathrm{C}$ to recover the catalyst. The resultant solution was analysed using gas chromatography (GC) with a Shimadzu GC-2014 GC equipped with an Inert CAP 1 column $(0.25$ mm I.D. $\times 60$ m length, GL Sciences). Time courses of the conversions, the yields, and the selectivities for the reactions of nitorobenzene using Pd/Si catalysts (1st-4th cycles) were shown in Figure S12-14.

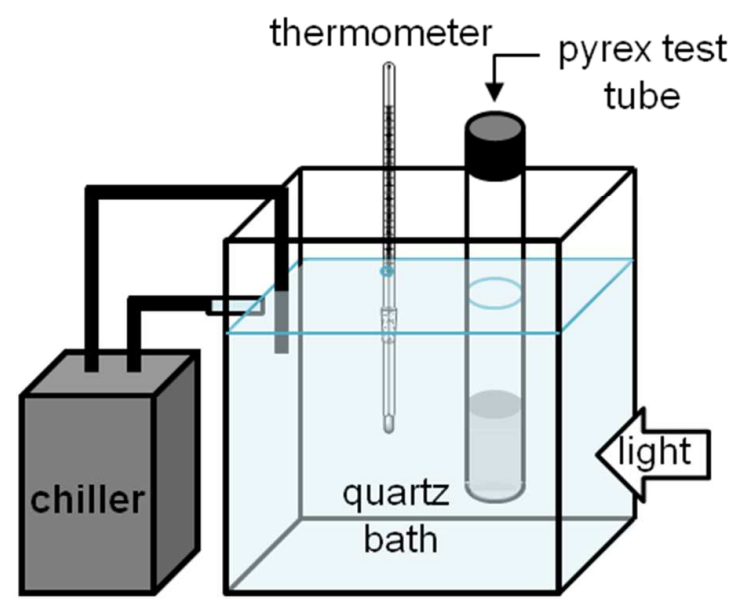

Figure S11. Schematic diagram of the photoirradiation reaction system. 


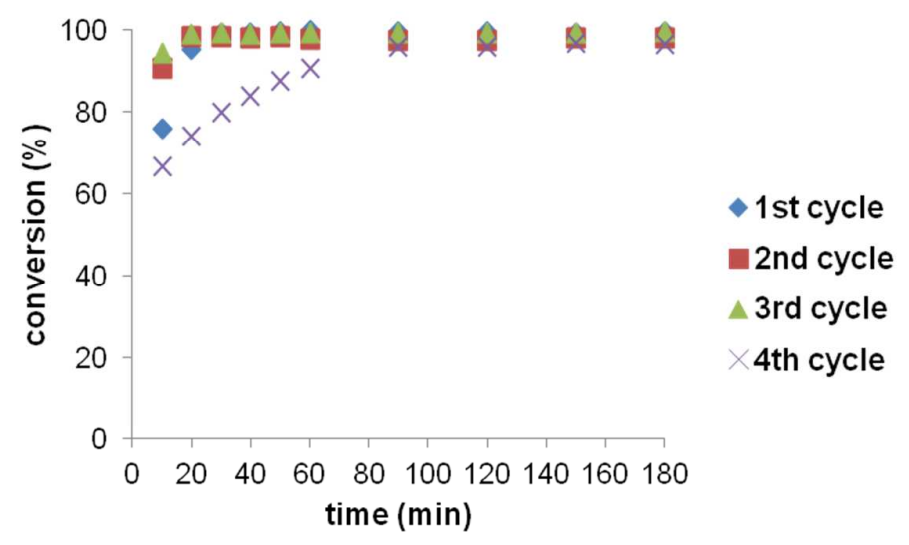

Figure S12. Time courses of the conversions of 1a.

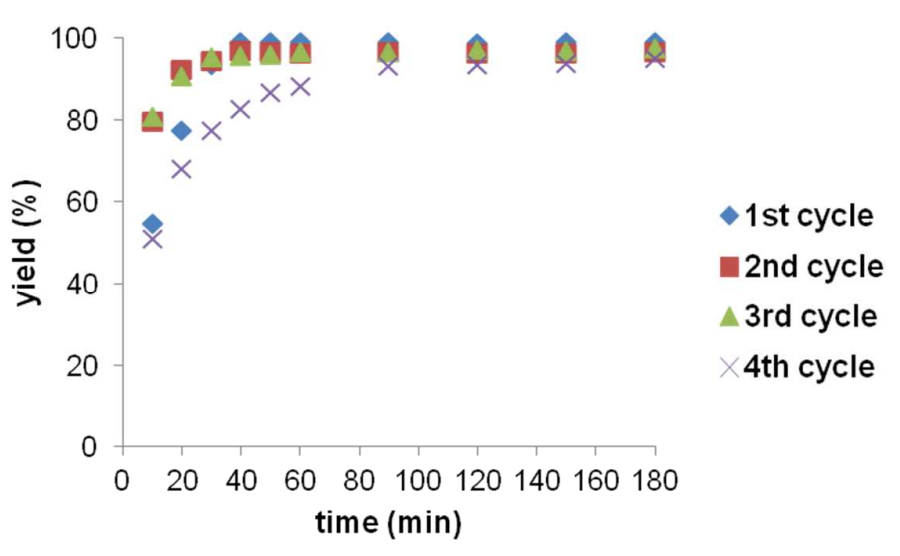

Figure S13. Time courses of the yields of $2 a$.

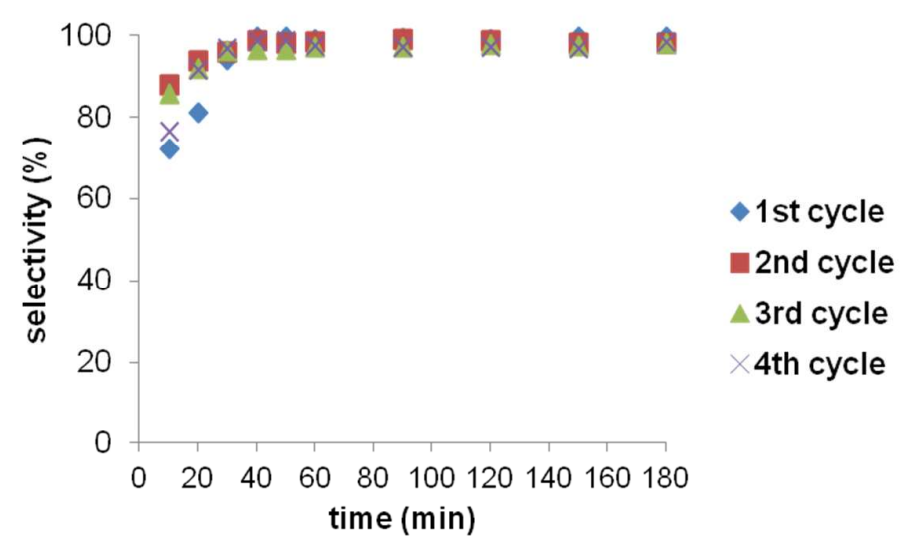

Figure S14. Time courses of the selectivities of $\mathbf{2 a}$. 